Supporting information

\title{
Effects of Regioregularity and Molecular Weight on the Growth of Polythiophene Nanofibrils and Mixes of Short and Long Nanofibrils To Enhance the Hole Transport
}

${ }^{1}$ Department of Materials Science and Engineering, Yonsei University, 50 Yonsei-Ro, Seodaemun-Gu, Seoul, Korea 120-749

${ }^{2}$ Research Institute of Iron and Steel Technology, Yonsei University, Seoul 120-749, Republic of Korea

${ }^{3}$ Department of Chemical Engineering, Pohang University of Science and Technology (POSTECH), 77 Cheongam-Ro, Nam-Gu, Pohang, Gyeongbuk, Korea 790-784

${ }^{4}$ Department of Materials Science and Engineering, Pohang University of Science and Technology (POSTECH), 77 Cheongam-Ro, Nam-Gu, Pohang, Gyeongbuk, Korea 790-784

${ }^{\dagger}$ The authors contributed equally to this work.

¥ Current address: Department of Chemical Engineering, Stanford University, California 94305, USA

*Corresponding authors: ujeong@ postech.ac.kr, taihopark@postech.ac.kr 

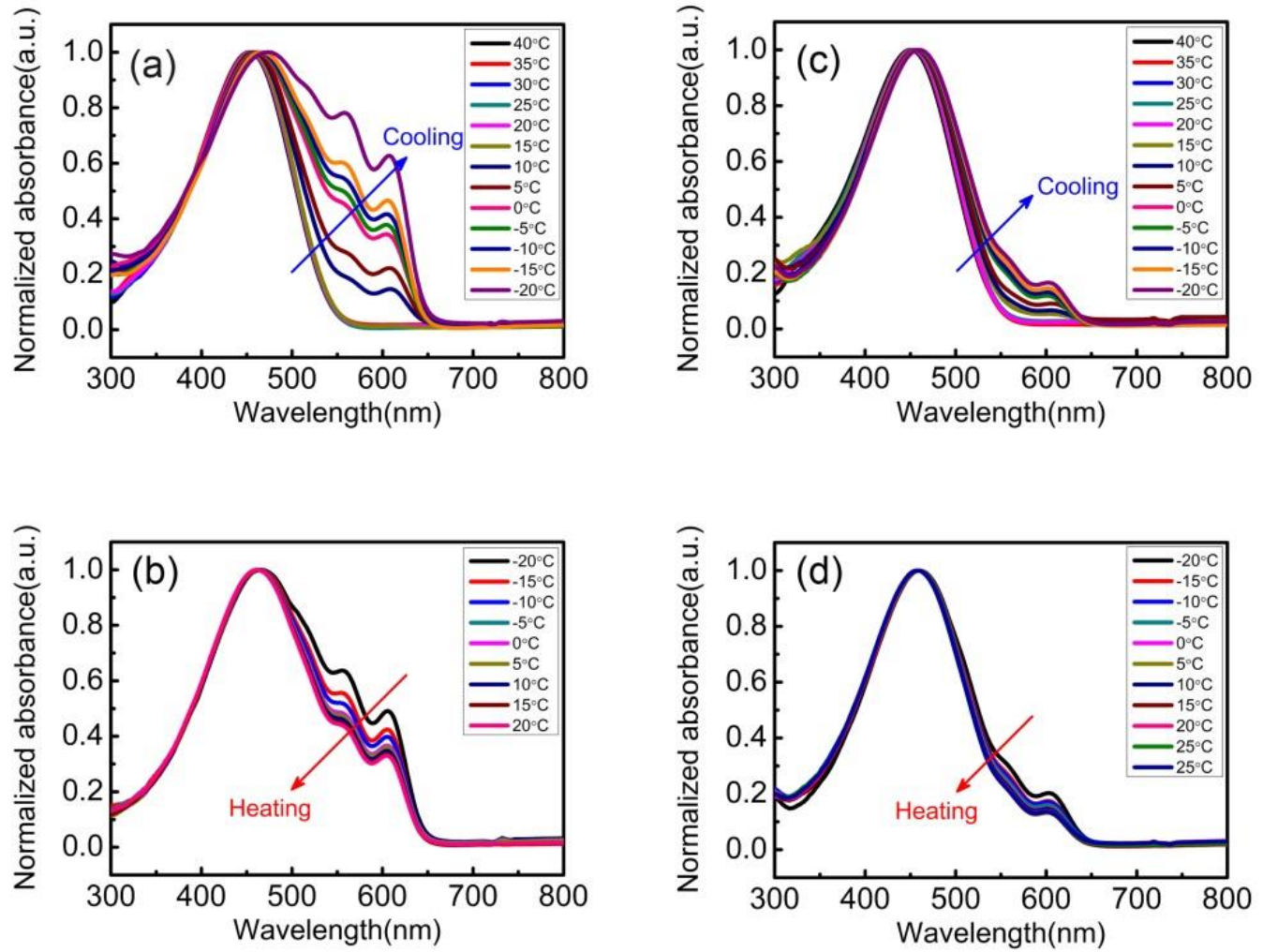

Figure S1. UV-vis absorption spectra of P3HTs in m-xylene solution during cooling to $-20^{\circ} \mathrm{C}$ and re-heating to $25^{\circ} \mathrm{C}$. The cooling and heating rates were $-8{ }^{\circ} \mathrm{C} / \mathrm{min}$ and $2{ }^{\circ} \mathrm{C} / \mathrm{min}$. The molecular weights of P3HTs were $15.3 \mathrm{kDa}(\mathrm{a}, \mathrm{b})$ and $12.7 \mathrm{kDa}(\mathrm{c}, \mathrm{d})$. 

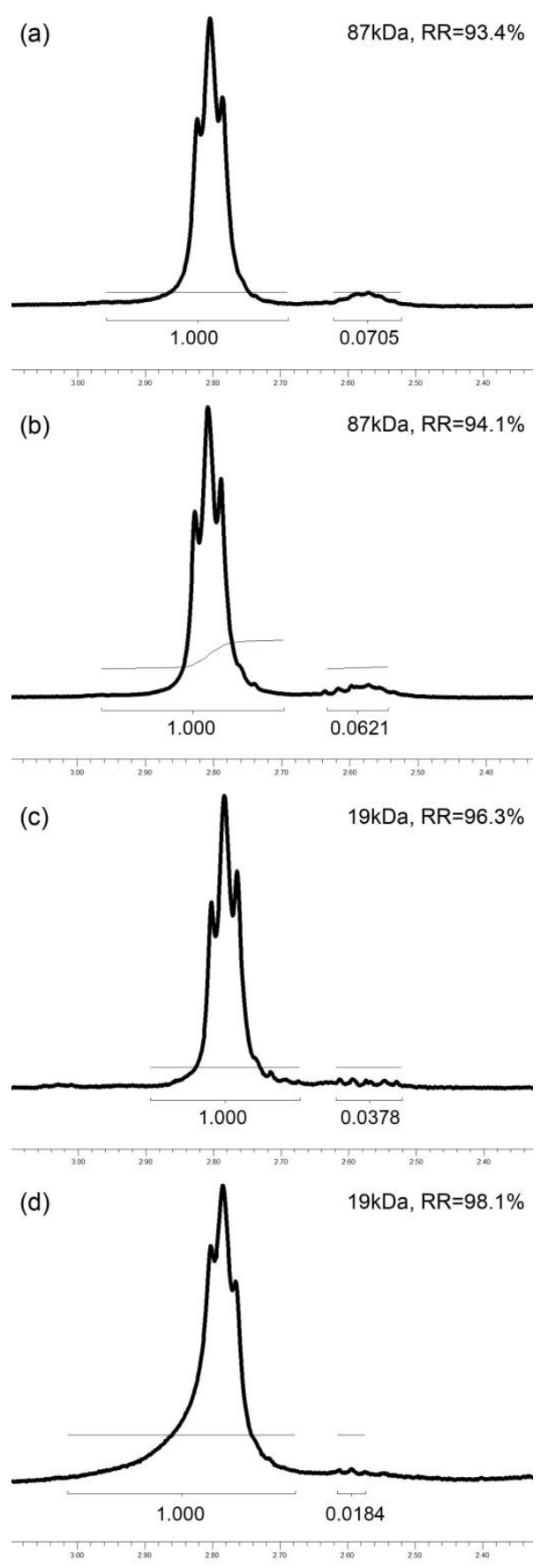

Figure S2. The change of ${ }^{1} \mathrm{H}-\mathrm{NMR}$ spectra of P3HT which were obtained before and after selectively extracting the high regioregular P3HT via centrifugation. The spectra of (a) and (b) were low-RR and high-RR 87kDa P3HT, respectively. The spectra (c) and (d) were low-RR and high-RR $19 \mathrm{kDa}$ P3HT, respectively. 
(a)

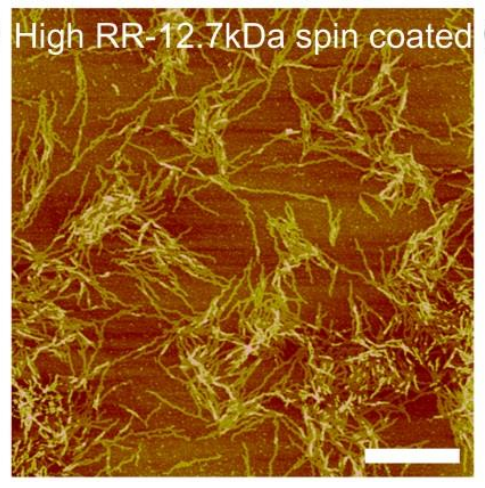

(b)

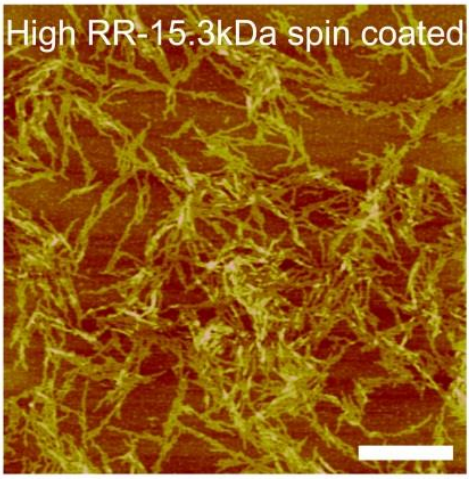

(c) High RR-19.7kDa spincoated

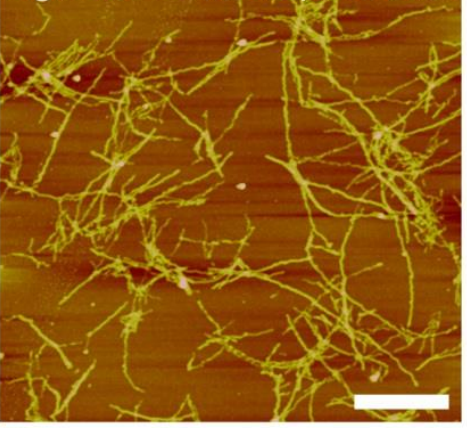

Figure S3. AFM images (tapping mode) of P3HT nanofibrils prepared by spin-coating high-RR P3HTs: (a) $12.7 \mathrm{kDa}$ with $96.9 \%-\mathrm{RR}$, (b) $15.3 \mathrm{kDa}$ with $96.9 \%-\mathrm{RR}$, and (c) $19.7 \mathrm{kDa}$ with $98.0 \%$ -RR. The high regioregularity was achieved by selectively extracting the highly regioregular polymers from the solutions. The scale bar in each figure is $1 \mu \mathrm{m}$. 

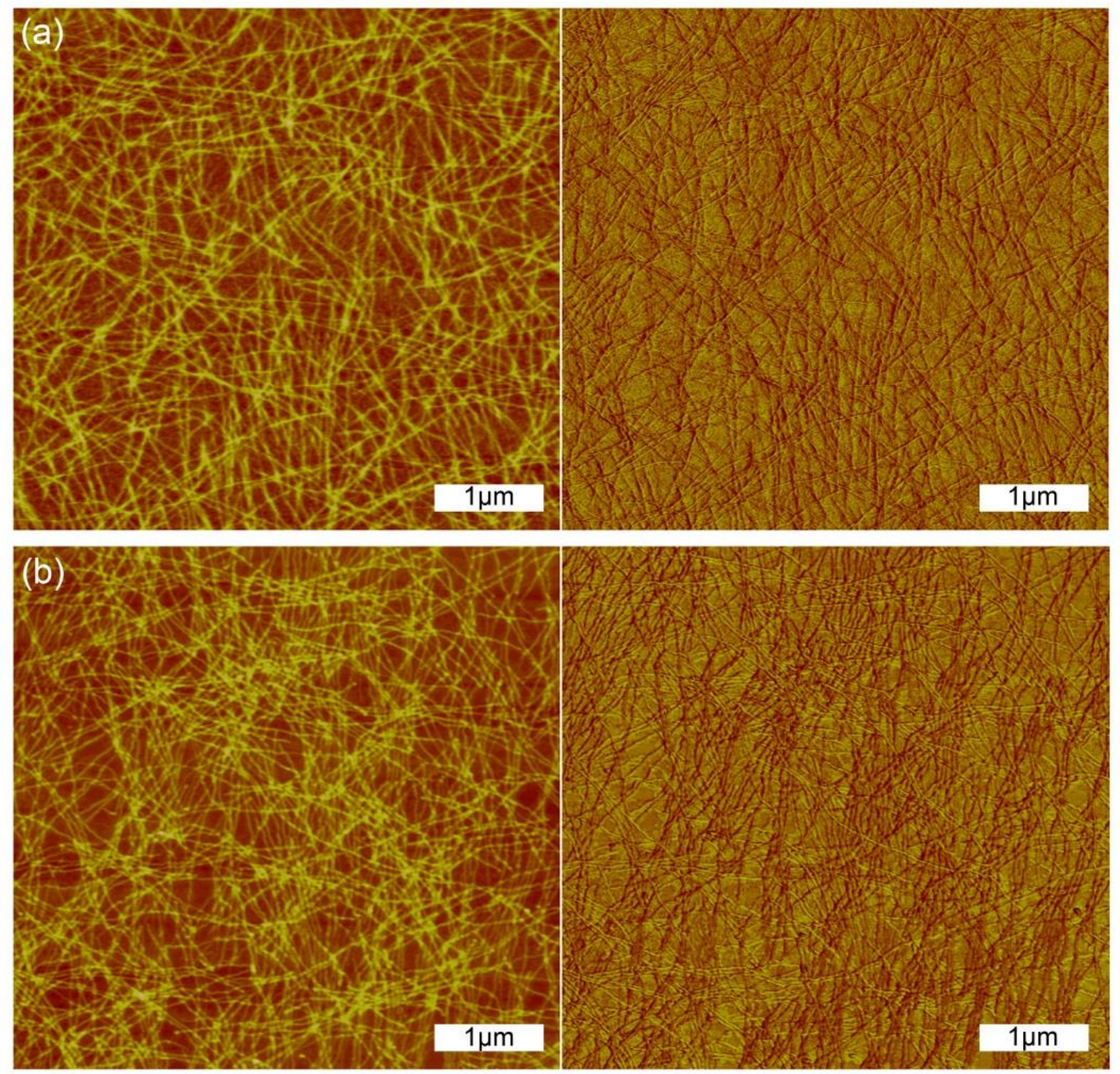

Figure S4. Height and phase AFM images of low-RR 87 kDa P3HT (a) and low-RR 19 kDa P3HT (96\%) (b) after washing out the possible impurities in the polymers. Long nanofibrils were formed in both cases, which indicates that removing the possible impurities is not the reason why the short nanofibrils were obtained in the high-RR counterparts. 

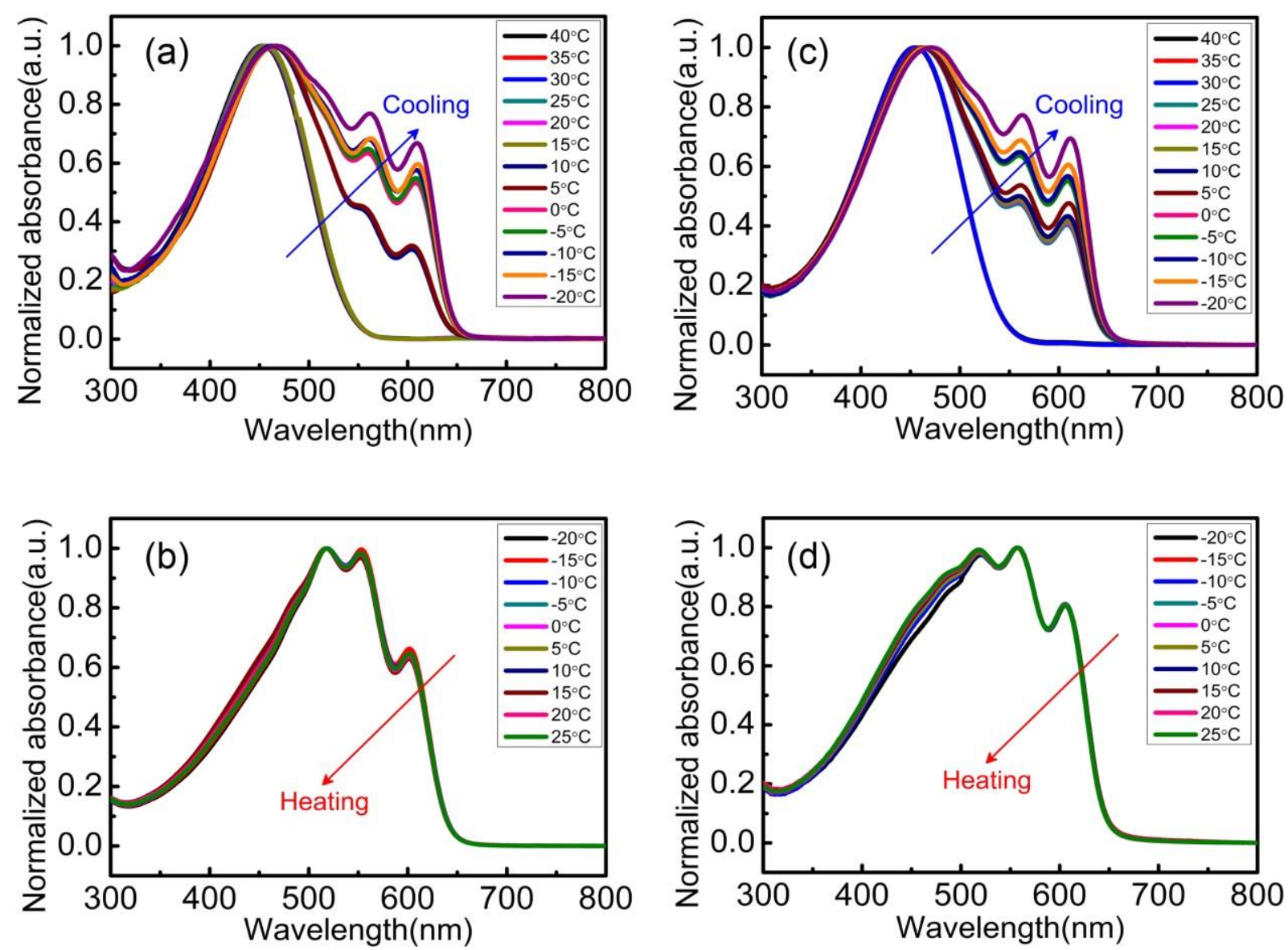

Figure S5. UV-vis absorption spectra of P3HTs in $m$-xylene solution during cooling and heating process for two different regioregularities having same molecular weight as $19 \mathrm{kDa}$. The regioregularities of $\mathrm{P} 3 \mathrm{HT}$ were $96 \%(\mathrm{a}, \mathrm{b})$ and $98 \%(\mathrm{c}, \mathrm{d})$. 

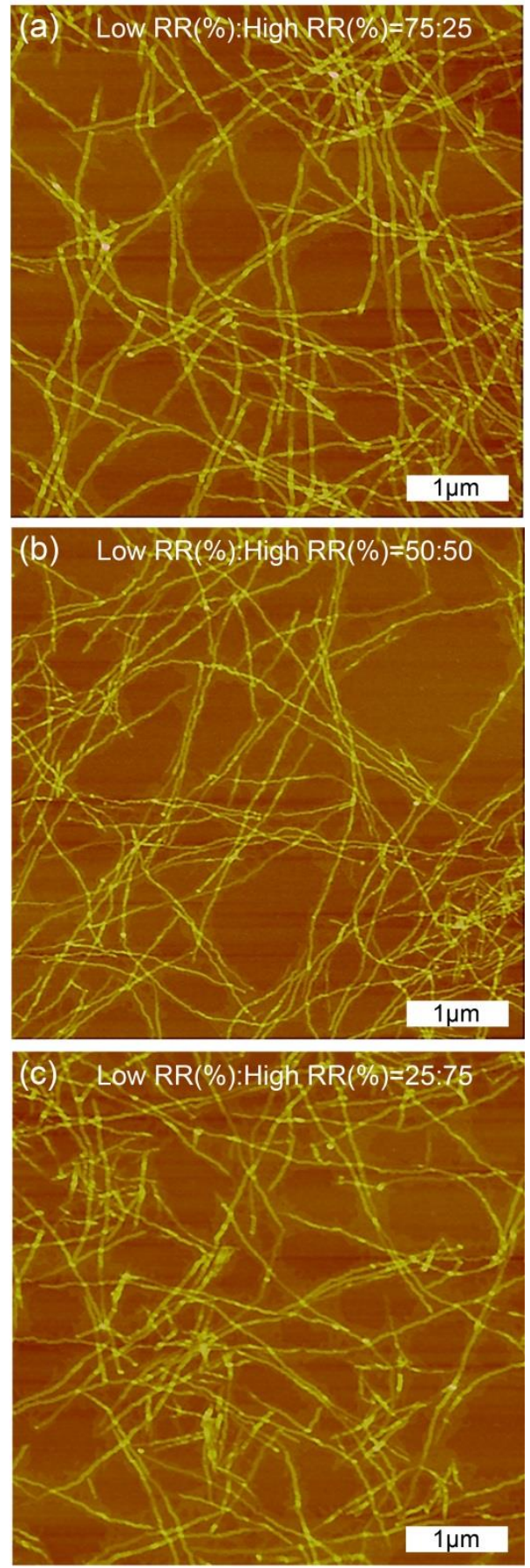

Figure S6. SEM images used to analyze the length distributions of the nanofibrils in the mixture films with different mixing ratios (low RR (87 kDa):high RR (50 kDa)). The mixing ratios were (a) 75:25, (b) 50:50, and (c) 25:75. 